\title{
The clincal characteristics of abdominal migraine and risk factors for developing migraine later in childhood
}

\author{
KH Lee*, EY Kim, JH Lee, SK Kim \\ From The European Headache and Migraine Trust International Congress \\ London, UK. 20-23 September 2012
}

\section{Objective}

Abdominal migraine (AM) is an idiopathic recurrent disorder occurring primarily in children. Because their abdominal pain may be so intensive to interfere with their normal activity and AM is recognized as migraine prodrome, it is important to make the exact diagnosis, appropriate managements and close follow-up the symptoms. We therefore analyzed the clinical characteristics of AM and risk factors for developing migraine later.

\section{Methods}

The 923 children with recurrent abdominal pain (RAP) visited our hospital from Jan 2006 to Dec 2010. Among them we retrospectively studied 84 children fulfilled ICHD-II criteria for AM. Through chart review and telephone interview, we evaluated the clinical characteristics of $\mathrm{AM}$ and divided them into two groups by developing for migraine (group A) or not (group B). By comparing the groups, we tried to find the risk factors for developing migraine later.

\section{Results}

About $8.9 \%$ of patients with RAP were diagnosed as AM. Their mean age was $7.1 \pm 3.0$ years (boys, $24,28.6 \%$; girls, $60,71.4 \%$ ). The frequency of abdominal pain was $4.3 \pm 2.4 /$ week and the duration of abdominal pain was about one hour (0.5 2.0). The symptoms associated with abdominal pain were anorexia $(n=35,15.5 \%)$, nausea $(n=58,69.0 \%)$, vomiting $(\mathrm{n}=26,31 \%)$, pallor $(\mathrm{n}=12,14.3 \%)$ and headache $(n=64,76.2 \%) .27$ (32.1\%) patients with AM were suffer from migraine $1.7 \pm 0.8$ years later from onset of $A M$, and their mean age was $8.3 \pm 3.2$ years (boys, $8,29.6 \%$; girls, 19 ,
70.4\%). When the clinical characteristics were compared to each other between group A $(n=27)$ and group $B(n=$ 57), there were no differences in age, gender, frequency, duration etc. But AM patients with headache significantly developed migraine later $(P=0.003)$. AM patients who needed drug therapy significantly developed migraine later $(\mathrm{P}=0.034)$.

\section{Conclusion}

$32.1 \%$ of AM patients developed migraine. And the risk factors for developing migraine were headache associated with AM and general impression by physician that need pharmacologic therapy.

Published: 21 February 2013

\section{References}

1. Abu-Arafeh I, Razak S, Sivaraman B, Graham C: Prevalence of headache and migraine in children and adolescents: A systematic review of population-based studies. Dev Med Child Neurol 2010, 52:1088-97.

2. Blau JN, MacGregor EA: Is abdominal pain a feature of adult migraine? Headache 1995, 35:207-9.

3. Bugdayci R, Ozge A, Sasmaz T, Kurt AO, Kaleagasi H, Karakelle A, et al: Prevalence and factors affecting headache in turkish schoolchildren. Pediatr Int 2005, 47:316-22.

doi:10.1186/1129-2377-14-S1-P4

Cite this article as: Lee et al:: The clincal characteristics of abdominal migraine and risk factors for developing migraine later in childhood. The Journal of Headache and Pain 2013 14(Suppl 1):P4. 